\title{
México desea mostrar a las naciones hermanas su vida intelectual: Gabriela Mistral y los escritores mexicanos 1916-1922
}

\author{
Mexico wishes to show its intellectual life to the sister nations: \\ Gabriela Mistral and the Mexican writers (1916-1922)
}

\author{
CARLA ULLOA INOSTROZA \\ Universidad Nacional Autónoma de México. \\ Correo electrónico: carlaulloa@filos.unam.mx
}

Una de las etapas más importantes en la trayectoria y biografía intelectual de Gabriela Mistral fue la vivida durante su residencia en México (1922-1924). Este artículo busca indagar cómo se produjo el acercamiento de la escritora chilena con sus pares mexicanos, durante los seis años previos a su viaje, para ofrecer una hipótesis al respecto; su exitoso mecanismo de acercamiento tuvo como consecuencia una inserción y participación exitosa en el campo cultural debido al contacto con la red de escritores centrales del periodo posrevolucionario mexicano; mayoritariamente enlazados al proyecto vasconcelista, quienes ocupaban importantes sitios de decisión y gestión en los aparatos de administración del Estado, en instituciones culturales y diplomáticas. El capital simbólico de la chilena se amplió y fortaleció dentro del campo cultural mexicano a propósito de sus alianzas, proceso también favorecido por el reconocimiento que esos agentes le otorgaron por su trabajo como editora y autora de Lecturas para mujeres, antología que repercutió en su trayectoria posterior (fortalecida y favorecida por la experiencia mexicana).

Palabras claves: Gabriela Mistral, México, SEP, escritores mexicanos.

This article explores an important stage in the intellectual biography of Gabriela Mistral: her residence in Mexico (1922-1924). I focus on how Mistral approached to Mexican intellectuals before her travel to Mexico, which resulted in her appointment to the Secretary of Public Education. I propose that this approach enabled her successful insertion to the cultural field of post-revolutionary Mexico, particularly through relationships with central Mexican writers -linked to José Vasconcelos- that occupied important positions in the State apparatus as well as cultural and diplomatic institutions. Mistral's symbolic capital was thus strengthened, as can been noticed in her role of editor and author of Readings for Women (1923) for the Secretary of Public Education, a fundamental book

\footnotetext{
${ }^{1}$ Esta investigación se desarrolló durante los años 2014-2018 en el marco de la tesis doctoral "La construcción de una intelectual: Gabriela Mistral en el campo cultural mexicano", financiada por el Consejo Nacional de Ciencia y Tecnología de México y la Universidad Nacional Autónoma de México.
} 
in her career. In this regard, I conclude that the Mexican years of Gabriela Mistral enabled the internationalization of her work.

Key words: Gabriela Mistral, Mexico, SEP, mexican writers.

\section{INTRODUCCIÓN}

"México acaba de enviar a los países sudamericanos una Embajada intelectual; México desea mostrar a las naciones hermanas su vida intelectual, porque no le conocemos sino sus desgracias políticas"; advertía Gabriela Mistral a los lectores chilenos el 26 de agosto de 1921 en El Mercurio, once meses antes de trasladarse a aquel país para trabajar en la Secretaría de Educación Pública (SEP). ¿Cómo se produjo la relación con México, sus escritores y publicaciones? ¿Es posible rastrear el inicio de la afinidad con ese país en las relaciones profesionales establecidas con autores y editores? ¿Cuáles fueron los autores con quien se relacionó antes del viaje? Y finalmente, ¿por qué México? Este artículo busca complejizar la investigación histórica sobre las relaciones bibliográficas e intelectuales de Gabriela Mistral con escritores mexicanos en el periodo que abarca los ańos 1916-1921, con el objetivo de explicar las circunstancias que posibilitaron su salida de Chile.

Varias veces se ha reparado en la importancia de México -como espacio de formación, aprendizaje y consolidación- en la vida intelectual de Gabriela Mistral desde la señera recopilación que efectuó el poeta Alfonso Calderón en Croquis mexicanos (1979) ampliada por Pedro Pablo Zegers en Gabriela y México (2007), destacando los aportes teórico-metodológicos de Elizabeth Horan en sus diferentes investigaciones, especialmente en Motivos: The life of St. Francis (2013), y los de Claudia Cabello Hutt en Artesana de si misma: Gabriela Mistral, una intelectual en cuerpo palabra (2018). A pesar de estos importantes avances, la relación de Gabriela Mistral con México sigue estando poco clara para el público general e inclusive para lectores enterados de la obra mistraliana, como el editor y crítico Cristián Warnken, que ha insistido en la idea de que "el ministro Vasconcelos se la lleva a México" restando agencia a la capacidad autogestiva de la poeta. Por esto, parece central revisitar el periodo a la luz de una investigación histórica que prepondere los hallazgos para posibilitar materiales significativos para el campo de estudios mistralianos y para la construcción de una historia de las relaciones intelectuales entre Chile y México.

Varios hechos me permiten justificar la significancia de la estadía en México como un periodo de profesionalización para Mistral; en primer lugar, durante esos meses la poeta logró publicar sus dos primeros libros: el poemario Desolación y la antología Lecturas para mujeres, consiguiendo además el contrato para publicar su segundo poemario Ternura en España y efectuando la redacción de Motivos de San Francisco un proyecto que finalmente se publicó póstumo. En segundo lugar, durante este tiempo participó en diversas instancias 
de sociabilidad con escritores mexicanos y extranjeros residentes en Ciudad de México desarrollando un habitus ${ }^{2}$ diferente al de maestra. En este país la chilena experimentó una ampliación de su capital simbólico ${ }^{3}$ y el posicionamiento como autora con alcance continental, estableciendo vínculos profesionales con hombres de letras en un contexto androcéntrico hetero-patriarcal que restringía dichas participaciones a las escritoras pero que presentaba las particularidades de un periodo posrevolucionario. Y, en tercer lugar, el gobierno de México a través del presidente Álvaro Obregón otorgó el financiamiento para su primer viaje a Estados Unidos y Europa, continente en donde se estableció por casi quince años gracias, entre otras cosas, al apoyo brindado por la intelectualidad ligada a la diplomacia y el gobierno mexicano, como defensora de diversas causas en especial la latinoamericana.

Sabemos gracias a los estudios mistralianos que la poeta contaba con al menos quince años de experiencia en trabajos complejos del ámbito de la educación chilena rural y urbana (en las ciudades de La Serena, Traiguén, Antofagasta, Los Andes, Punta Arenas, Temuco y Santiago), antes de su traslado a México. La profesora había realizado prácticas en labores sociales activistas a favor de obreros y campesinos; mientras ejercía la crítica literaria y un intenso intercambio epistolar con escritores latinoamericanos. Además, publica su poesía y prosa en diversos países como, por ejemplo, México. La excelente formación autodidacta de la poeta en historia y literatura durante su juventud (1889-1921) se vio favorecida por su ejercicio como profesora (desde 1904) primero, en pequeñas escuelas rurales, y luego como docente y directora de Liceos de Nińas en distintas ciudades chilenas; trabajos que le brindaron experiencia con organismos burocráticos de instrucción y educación pública, entrenándose en iniciativas políticas locales para la mejora de las condiciones de vida de indígenas, trabajadores y campesinos; también en la vinculación permanente a funcionarios públicos, escritores y grupos de avanzada comprometidos con el cambio social a favor de las mayorías subordinadas. En ese sentido, sus escritos del periodo chileno (como, por ejemplo, el ensayo "El Grito") lograron condensar preocupaciones por la justicia social que también ocupaban a sus pares mexicanos, sintonizando con ciertos intereses y objetivos propuestos por el orden simbólico posrevolucionario mexicano.

Gabriela Mistral desarrolló en su etapa chilena un incipiente pensamiento latinoamericanista, arraigado en una experiencia de clase (trabajadora, campesino-rural) y

\footnotetext{
${ }^{2}$ Concepto del sociólogo francés Pierre Bourdieu que hace referencia a la disposición de los esquemas culturales y de época mediante los cuales los sujetos perciben el mundo y se desenvuelven en él, en este caso, la forma de relaciones sociales de los escritores del momento, fuertemente marcada por una homosociabilidad. Para explicaciones sobre este concepto y el de "campo"; véase Bourdieu 2002.

${ }^{3}$ Siguiendo con Bourdieu; a diferencia de la acumulación de capital económico que persigue la adquisición de bienes materiales, la acumulación de capital simbólico se produce cuando el creador del bien simbólico recibe legitimidad y reconocimiento, cuando se le acepta como un productor habilitado para emitir esos bienes culturales.
} 
una sólida autoformación literaria, que queda de manifiesto en su obra ensayística anterior a 1922. En ese contexto, la poeta recibió a fines de 1921 o principios de 1922 la invitación de José Vasconcelos para trasladarse a México y trabajar en SEP. Las desafiantes experiencias vividas por Gabriela Mistral en Chile, sin embargo, estaban limitadas. Su llegada a México -en julio de 1922, con 33 años de edad- se producía como una evidente desconocida, aunque contando con el reconocimiento brindado por el círculo de hombres de letras que la recibía: los principales escritores mexicanos vivos y relacionados con José Vasconcelos habían leído su obra y esperaban su llegada como invitada de honor de la SEP y del gobierno posrevolucionario del general Álvaro Obregón.

José Vasconcelos, que lideraba en ese momento una reforma educativa inédita invitó a Gabriela Mistral con un "augurio de dicha". Su estadía tuvo como principales consecuencias la dedicación exclusiva a la lectura y escritura, la experiencia de un cuarto propio $o^{5}$ que permitió lucir sus talentos gracias a la seguridad económica y el respaldo a su quehacer brindado por el estado mexicano y alcanzar una productividad impresionante. He mencionado al inicio los poemarios y la antología que Mistral redactó en México, un volumen que se acerca al tercio de su obra total (excluyendo por cierto la prosa). Sostengo entonces que la trayectoria de la poeta se densifica en estos intensos ańos de aprendizaje y a su vez ejecuta nociones antiguas dentro de sus intereses (1922-1924), en sintonía con las preocupaciones por la justicia social devenida de los ideales arielistas de la Generación del Ateneo ${ }^{6}$ y del círculo vasconcelista.

Mistral fungió como un sujeto intelectual multifuncional en México; por un lado, trabajó con José Vasconcelos en instancias específicas de la SEP, con la instalación de una escuela modelo rural, la implementación de bibliotecas públicas, visitas a escuelas indígenas, la edición del libro Lecturas para Mujeres y el proyecto colectivo de Lecturas clásicas para niños. Además, conferencista y visitante de escuelas en diversos estados del país como figura homenajeada, portadora de mensajes de estudiantes y obreros chilenos ante sus pares mexicanos, como miembro de asociaciones mexicanas (Congreso de Maestros Misioneros, Congreso del Niño, Acción Iberoamericana) y mediadora entre las embajadas de Chile y México; pero principalmente como promotora y propagandista del gobierno de Álvaro Obregón (Ulloa 2017), en momentos donde los ataques de Estados Unidos significaban el bloqueo de su vecino sureño. Estos trabajos le merecieron una comisión auspiciada en Europa financiada por el estado mexicano, asunto central considerando la precarización constante de su participación ad honorem en los consulados chilenos.

\footnotetext{
${ }^{4}$ Para un exhaustivo análisis de la gestión de José Vasconcelos en la SEP véase Fell 1989.

${ }^{5}$ La expresión la tomo de $A$ room of onés own, libro de Virginia Woolf publicado en 1929, cuyo objetivo fue abogar por un mayor espacio literal y ficticio para las escritoras ofreciendo una noción de intelectual diferente a la que pensó simultáneamente Antonio Gramsci.

${ }^{6}$ Para más detalles del arielismo mexicano véase Luigi 2016.
} 


\section{VÍNCULo PREVIOS AL VIAJE (1916-1921)}

Cuadro 1. Publicaciones de Gabriela Mistral en México antes de su estadía (Ulloa 2019: 115-116).

\begin{tabular}{|c|c|c|c|}
\hline Año & Editores & Revista & Colaboraciones de G. Mistral \\
\hline 1917 & $\begin{array}{l}\text { Enrique González Martínez } \\
\text { Ramón López Velarde } \\
\text { Efrén Rebolledo }\end{array}$ & Pegaso & $\begin{array}{l}\text { "Las manos cobardes" } \\
\text { "Mi corazón" } \\
\text { "A la discípula" }\end{array}$ \\
\hline 1920 & $\begin{array}{l}\text { Enrique González Martínez } \\
\text { Genaro Estrada }\end{array}$ & $\begin{array}{l}\text { México Moderno. Revista } \\
\text { mensual de Letras y Arte }\end{array}$ & $\begin{array}{l}\text { "Decálogo del artista" } \\
\text { *"La misión de Antonio Caso" } \\
\text { (1921) }\end{array}$ \\
\hline 1921 & Excélsior & Revista de Revistas & $\begin{array}{l}\text { "Mujeres en la Biblia: Ruth" } \\
\text { "El grito" } \\
\text { * "A González Martínez" }\end{array}$ \\
\hline 1921 & $\begin{array}{l}\text { José Vasconcelos } \\
\text { Enrique Monteverde } \\
\text { Agustín Loera y Chávez }\end{array}$ & $\begin{array}{l}\text { El Maestro: Revista de } \\
\text { Cultura Nacional }\end{array}$ & $\begin{array}{l}\text { "El grito" } \\
\text { "Una carta a José Vasconcelos" } \\
\text { "Piececitos" } \\
\text { "Himno al árbol" } \\
\text { "El poema de la madre" } \\
\text { Lecturas escolares: "La charca" y } \\
\text { "El cardo" } \\
\text { Rondas de niños: "En donde } \\
\text { tejemos la ronda", "La margarita", } \\
\text { "Invitación”, "Dame la mano", } \\
\text { "Los que no danzan", "La tierra", } \\
\text { "Jesús" y "Todo es ronda" }\end{array}$ \\
\hline 1921 & $\begin{array}{l}\text { Departamento de } \\
\text { Bibliotecas de la SEP }\end{array}$ & El Libro y El Pueblo & $\begin{array}{l}\text { *Páginas inéditas de Gabriela } \\
\text { Mistral: "El Canto" }\end{array}$ \\
\hline
\end{tabular}

*Indica que fueron publicados cuando la autora ya vivía en México, pero fueron escritos y enviados desde Chile.

El Cuadro 1 permite constatar a simple vista, algunos hechos. Primero: El Maestro fue la revista que concentró la participación de Gabriela Mistral antes de su viaje a México. Si consideramos que José Vasconcelos fue quien la invitó a viajar, el resultado parece evidente (El Maestro fue también la revista en la que más publicó durante los veintiún meses de su permanencia en México). En segundo lugar, resulta fácil constatar que publicó más durante los meses previos a su viaje, seguramente ante la necesidad de darse a conocer al público mexicano ya que sin libros publicados su nombre aún era poco familiar. Es destacable señalar que los inicios de la década de 1920 han sido identificados como momentos propicios en el campo periodístico y editorial mexicano en tiempos donde los escritores -en 
especial los poetas- ocupaban espacios claves en la dirección, edición y notas periodísticas (Sánchez-Prado 2006: 12). Como bien advierte Pedro Ángel Palou "la poesía seguía siendo considerada por los mismos agentes del campo, como el más alto género de las letras" (Palou 1997: 83). Otro aspecto que debemos atender, en tercer lugar, son los protagonistas de las colaboraciones enviadas y publicadas por Gabriela Mistral en México con anterioridad a su establecimiento en el país. Figuran los escritores que ella conoció personalmente en Chile; Antonio Caso ${ }^{7}$, Enrique González Martínez, Vasconcelos su amigo epistolar, y a quien admiraba; María Enriqueta Camarillo. Finalmente, en cuarto lugar, la única colaboración que se repite es el ensayo "El Grito", según mi perspectiva pieza arielista fundamental en su alianza con el proyecto vasconcelista.

A la hora de reconstruir el acercamiento de Gabriela Mistral con el campo cultural mexicano también debemos tener presente el epistolario con el poeta Amado Nervo, con quien mantuvo correspondencia desde 1916. Nervo, como escritor afamado y reconocido en su país desde fines del siglo XIX, participó en diversas publicaciones de gran importancia para la literatura mexicana del periodo; Revista Azul y Revista Moderna, entre otras. No obstante, su temprano fallecimiento en $1919^{8}$ impidió el desarrollo de un vínculo mayor entre la chilena y el mexicano. Probablemente el conocimiento que Mistral tuvo de las revistas mexicanas inició ese año ya que al rastrear las diferentes publicaciones de las que participaba Nervo aparecían autores de la generación del Ateneo de la Juventud. Por ejemplo, Revista $A z u l$ (1894-1896) que fue publicada semanalmente en Ciudad de México, era dirigida por el poeta Manuel Gutiérrez Nájera, portavoz del modernismo en América Latina; en ella se daba a conocer la poesía europea de Baudelaire y Verlaine, por una parte, y la latinoamericana de Rubén Darío, José Santos Chocano, José Martí, Salvador Díaz Mirón, Amado Nervo, Manuel José Othón, María Enriqueta Camarillo, José Juan Tablada, Luis Gonzaga Urbina, entre otros. Quiero apuntar al hecho de que todos estos autores que selecciono (excepto Verlaine) fueron incluidos por Gabriela Mistral en Lecturas para mujeres.

Cuando la poeta llevaba unas semanas en Ciudad de México reforzó la admiración e identificación que sentía con la obra de Amado Nervo, visitando su tumba para dejarse tomar fotografías que acompañaron la publicación de noviembre de 1922; un emotivo ensayo titulado "A Amado Nervo" (Zegers 2007: 51-52), en el que señalaba: "tus cartas atravesaban el mar para ir a buscarme"; "viviendo yo ahora entre tus gentes tú me llevas de la mano, tu nombre me es amigo desde la sepultura".

Con Nervo, Mistral sentía una fuerte y genuina conexión estética:

\footnotetext{
${ }^{7}$ Antonio Caso fue rector de la Universidad Nacional en el periodo 1921-1923, según he rastreado en la prensa de la época visitó Santiago de Chile en julio de 1921, oportunidad en donde conoció a Gabriela Mistral. Fue noticia la invitación que la poeta le cursó al rector pues la conferencia en el liceo que dirigía quedó plasmada en el ensayo "La misión de Antonio Caso"; (véase Zegers 2007: 44-45).

${ }^{8}$ Cuando Nervo murió Mistral publicó el poema "In memoriam" en su honor, en Revista Mireya (1919) que fundó ese año junto a Julio Munizaga, con seis números publicados entre mayo y noviembre. En sus páginas la poeta también publicó extractos de Desolación y aparecieron colaboraciones de Amado Nervo, Juana de Ibarbouru, Ramón del Valle Inclán y Alfonsina Storni, entre otros.
} 
¡Bendito seas! Trajiste a la América centauresca el canto suavizante; desdeñaste la fea espesura de la épica e hiciste de tu verso la levedad de un manojo de hierbas para que los tristes lo pusieran contra sus heridas. En vez de la dureza que tiene el ramo de la pińa americana, diste a la estrofa la suavidad de los helechos altos; hablaste con una voz que era menos que un suspiro, para aguzar los duros oídos de tu recia América.

Tu poesía sigue caminando por el Continente (Zegers 2007: 52).

Para el investigador Juan Loveluck esta sintonía se debió a que Mistral se sentía atraída debido a la inclinación teosófica del mexicano; les unía el "neoespiritualismo" de la época:

El prestigio esotérico -los famosos retratos de Nervo "con sortija" y cara de vidente o demiurgo- las devociones teosóficas y las confianzas metempsicósicas, la fuerte atracción por el más allá, los muchos años de cantar a una figura amada y muerta en plena juventud, el tagorismo epocal, que ella tuvo oportunidad de ver estimulado en Chile, con traducciones en las que colaboró con varias glosas (Loveluck 1970: 497).

Otro caso destacable es el vínculo establecido con Enrique González Martínez (1871-1952) quien fuera el primer escritor mexicano que Mistral conoció personalmente. El 17 de septiembre de 1920 le escribió a un profesor chileno:

Ha llegado a esa [ciudad] el doctor E. González Martínez, profesor cultísimo y poeta americano de alta valía. Yo deseo que usted vaya a verlo pronto. No solo los escritores, sino los maestros, deben hacerle grata su estadía en nuestra tierra. He hecho este pedido únicamente a usted y al Dr. F. Peña. Estimo mucho al mexicano y deseo verlo rodeado en mi país de la gente que yo amo y aprecio (Mistral 1920).

El nombre del embajador mexicano era conocido para Mistral, pero desde el punto de vista literario con relación al trabajo de González Martínez como poeta y editor, ya que justamente conocía su existencia desde Pegaso, primera revista literaria en la que Gabriela Mistral publicó en México (que había sido fundada por González Martínez, Ramón López Velarde y Efrén Rebolledo). Sobre el debut de la poeta en México Pegaso señaló:

Fieles a nuestro afán de difundir el conocimiento de los poetas que florecen en Hispano América, reproducimos hoy tres magníficos poemas de Gabriela Mistral. Las rimas audaces y escabrosas, junto a una sumisión suavemente femenina, producen un efecto asombroso. Esta alta poetisa chilena, era hasta hoy, desconocida en México (Pegaso 1917).

Dejando de lado el análisis que merece el desfavorable empleo del concepto sumisión, se incluyeron los poemas "Las manos cobardes", "Mi corazón" y "A la discípula". El afán 
hispanoamericanista de Pegaso, por cierto, ayudó al debut de Mistral en México, siendo este otro aspecto central ya que las ideas debatidas en el incierto periodo posrevolucionario y los afanes políticos de la escritura hicieron posible también la conexión entre la chilena y los mexicanos.

Esta revista, de publicación semanal, tuvo veinte números entre marzo y julio de 1917, en ella publicaron también Amado Nervo, Antonio Caso, Luis Gonzaga Urbina, Julio Torri, María Enriqueta Camarillo, Juan José Tablada, Alfonso Reyes, Genaro Estrada y Rubén Darío; todos ellos incluidos posteriormente en Lecturas para mujeres, lo que hace posible señalar que la chilena prestó atención a la revista y que tanto esta publicación como otras del periodo conformaron el crisol de nombres mexicanos que ella incluiría posteriormente en su antología.

Mistral y Enrique González Martínez se conocieron en Santiago, donde la poeta ocupó el cargo de directora del Liceo de Nińas y gozaba de reconocimiento por parte de un importante grupo de escritores chilenos; los que eran además conocidos por González Martínez ya que el embajador había reseñado la antología del colectivo Los Diez en Pegaso. Es importante señalar que Mistral tuvo diversas consideraciones con Enrique González Martínez en Chile, poniéndole en contacto con escritores y realizando una alianza en términos de difusión de los logros de la revolución mexicana, a través de conferencias y columnas en la prensa chilena. González Martínez fue central en el campo cultural mexicano de la primera mitad del siglo XX, tanta fue su importancia que al momento de su muerte fue velado en el Palacio de Bellas Artes y enterrado en la Rotonda de los Hombres Ilustres; de acuerdo con los testigos del funeral solo Amado Nervo había protagonizado exequias tan relevantes (Martínez 2002: 13), el enorme capital simbólico del mexicano no había sido adquirido únicamente por su calidad literaria sino también por su capacidad de gestión de publicaciones y su experiencia diplomática. Cuando Mistral vivió en México no contó con su presencia, ya que el poeta se encontraba aún en el extranjero (tal vez a modo de destierro debido a que no era escritor partidario de la revolución). De todas maneras, el vínculo entre ambos se fortaleció puesto que, en noviembre de 1923, al final del discurso en la Escuela Nacional Preparatoria, Mistral señaló:

Ha correspondido el discurso oficial de la fiesta, a un poeta joven de México. Recibís vos en él la simpatía de los nuevos que, como os he dicho, son muchos y de firmes valores. Para mí es grata doblemente su voz: pone aquí como la presencia de un querido ausente: el doctor González Martínez, quien me hizo deudora de México antes de que yo pisara su tierra. Quiero terminar con este recuerdo mi larga lectura. Hace tres años pedía yo, por primera vez, un ascenso a mi Gobierno: el Liceo número 6 de Santiago de Chile. Se trataba de un puesto disputadísimo. La primera carta que el presidente Alessandri recibió de mi favor fue la del ministro mexicano.

Su hijo ha querido asociarse a este acto por una razón especial: la admiración que él tiene hacia la muy altiva y muy noble juventud universitaria de Chile, y ha querido también estar con la maestra chilena en esta hora, como habría estado el ilustre ausente. Muchas veces, igracias! (Zegers 2007: 128). 
Los dos primeros escritores mexicanos con los que Gabriela Mistral entabló relación fueron Nervo y González Martínez, luego con Vasconcelos. A diferencia de los primeros dos poetas, Vasconcelos era escritor con un poder de acción más amplio, hábil y comprensivo de la escena continental y nacional. En lo concerniente a las instituciones, el primer secretario de la SEP había trazado un plan modernizador, práctico y en directo beneficio de su plan educativo: alfabetizar masivamente a la población y elevar la cultura. En sus propias palabras se trataba de "la aventura de regenerar a un pueblo por la escuela" (Vasconcelos 1968: 9). Creó tres grandes departamentos para gestionar la SEP: escuelas, bibliotecas y Bellas Artes, en la última incluyó a los muralistas, músicos, deportistas y a los museos. Como departamento auxiliar estableció las misiones de enseñanza indígena y alfabetización y creó los talleres tipográficos para proveer de libros a la SEP, fundando también bibliotecas que ofrecían espectáculos musicales, teatrales y conferencias, con el objetivo de afectar al público. Proyectó una SEP en manos de artistas e intelectuales, por ello se rodeó principalmente de poetas a quienes ubicó en puestos claves como jefes y administradores de departamentos, convocó a los pintores muralistas y protegió a intelectuales extranjeros que realizaron trabajos esporádicos y específicos como Gabriela Mistral. Con un profundo sentido práctico -y por cierto discutible desde múltiples sentidos- la idea del secretario era establecer una cultura superior, única, patriótica pero universal. Como activo agente del campo cultural buscaba crear un "estado de ánimo" con "poder de contagio" persuadiendo al público a través del arte: con conciertos, conferencias y mítines (Vasconcelos 1968: 10-11). El ex rector de la Universidad Nacional quería convencer al resto de la población, conquistar por medios de las ideas y sobre todo por el ejemplo, suyo y de los integrantes de su red como Mistral, a quien le manifestó; "es usted tan responsable de todo lo que ocurra mañana en México, como cualquiera de los que nacimos aquí mismo, en esta porción de la gran patria latinoamericana” (Zegers 2007: 27). Comprendía que el poder persuasivo radicaba también en la prensa, por eso se imprimía el Boletín de la SEP; sumario de las obras realizadas. A ese contexto organizacional llegó Gabriela Mistral con una capacidad de gestión imposible en el Chile de la "cuestión social". En 1921, Mistral publicó el pequeño ensayo "La cultura mexicana” cuyo principal objetivo era el de informar a los lectores chilenos sobre lo que sucedía en México ya que:

Siendo [Chile] un pueblo de vida política tranquila, juzgamos del progreso de los demás pueblos, únicamente por este aspecto y no nos explicamos que pueda existir, al margen de la situación interna agitada, una intensa actividad intelectual; no creemos que junto a los caudillos haya una legión, toda una legión de constructores de la cultura, labrándola con una serenidad y una confianza nobilísimas, desde la escuela primaria hasta la de Bellas Artes (Zegers 2007: 13).

$\mathrm{Al}$ comenzar su ensayo de esa manera la poeta apelaba a una función informativa de su escrito, en pos de la cultura general de Chile, país alejado y escasamente conectado con la realidad latinoamericana. Con un fuerte contenido interpretativo de la realidad de su época la autora prosiguió señalando: 
Yeste es el caso de México. Es toda una revelación ir conociéndolo por sus publicaciones, un movimiento pedagógico serio y firme, orientado según las ideas más modernas; un movimiento científico superior al nuestro y una obra literaria que alcanza la calidad de Argentina, y según algunos la sobrepuja [...]

Es triste, es hasta vergonzosa nuestra ignorancia de los valores intelectuales de la América en general y de México en particular. No hasta hace mucho llegaba a nuestras librerías el libro Estudios Indostánicos, de don José Vasconcelos, rector de la universidad mexicana. Se trata de un libro erudito y bello, rara alianza de una sabiduría y un temperamento emotivo, que nos hacía recordar la imagen de Víctor Hugo: la ligera, la transparente gota de agua que lleva en sí todas las profundidades del cielo y el mar sin perder su divina levedad, su clara belleza. El libro nos mostraba a un escritor de primera fila y buscamos otros libros suyos, y nos encontramos con Prometeo, maravillosa producción de un pensador que no olvida nunca la gracia, un Rodó que ignoramos y que admiraríamos desde la segunda página de su lectura (Zegers 2007: 42-43).

Con la notable comparación de Vasconcelos y Rodó, Mistral insistió en la promoción latinoamericanista, en un ensayo que le permitió mostrar su utilidad a los intereses de la SEP y del gobierno de Álvaro Obregón que buscaba un estrechamiento de lazos latinoamericanistas en medio de las tensiones internas y externas. Una segunda lectura posible de este ensayo está en sintonía con la propuesta analítica de la prosa periodística mistraliana que ha realizado la investigadora Claudia Cabello Hutt. Esta explica la alianza con intelectuales en el poder a través de la publicación de textos laudatorios que le permitía hacer público quiénes eran sus maestros y cuál era su escuela de pensamiento, también como punto de partida para relaciones intelectuales y de amistad que harán más visible a Mistral en el mapa cultural (Cabello 2018); asunto relevante si tenemos en cuenta que Mistral fue una escritora autodidacta que no cursó estudios universitarios ni perteneció a una generación en específico, ni se identificó con un movimiento literario. Esa notable independencia, indiferencia con las modas y falta de definición militante contrasta con el aspecto que ilumina Cabello Hutt: Mistral solía dedicar sus poemas, recados, crónicas y ensayos periodísticos.

Las recurrentes menciones a Nervo, Caso y González Martínez como paladines de la cultura a la revista México Moderno y a la editorial México como los mejores de América, creo son parte de una estrategia de posicionamiento más que un mensaje laudatorio, siguiendo a Pierre Bourdieu, ya que la alianza con estos escritores (y no otros) implicó de hecho un posicionamiento político en México. Los cuatro personajes que mencionó Mistral en este ensayo estaban involucrados con el aparato estatal posrevolucionario a través de la educación (Vasconcelos y Caso), o de la diplomacia (Nervo y González Martínez), como representantes de la intelectualidad en el poder. Los cuatro escritores además poseían una vasta lista de contactos con editoriales, poetas, políticos, académicos y colegas de todo el continente, ya que los cuatro fueron grandes viajeros. Mistral además les dedicó los poemas "In Memoriam" a Amado Nervo, "Ruth" a González Martínez (ambos aparecieron en Desolación) y el "Himno al Árbol" a José Vasconcelos (apareció en Tala). Para muestra otro ejemplo, en noviembre de 
1922, ya estando en México, escribió el ensayo "Una puerta colonial" (incluido en Lecturas para mujeres) dedicado a Alberto Pani, Secretario de Relaciones Exteriores durante ese año, contacto que le sería clave en su futura designación a Europa por parte del gobierno de Obregón y en su trabajo en Roma junto a Alfonso Reyes en el Instituto de Cooperación Intelectual (Reyes fue sucedido por Pani). En este sentido las habilidades autodidactas de Gabriela Mistral, sumadas a la capacidad de generar vínculos respetuosos, colaborativos, amistosos, solidarios, efectivos, incidentes y la correcta identificación del panorama político de su época, le permitieron tomar decisiones correctas para poder alcanzar una vida profesional en México mucho más amplia que la de una maestra.

\section{ESCRITORES MEXICANOS EN LECTURAS PARA MUJERES}

Todos estos vínculos que Mistral construyó desde Chile se materializaron cuando se estableció en México para realizar la principal tarea para la que fue contratada (con uno de los sueldos más altos de la SEP), es decir, la redacción de su antología Lecturas para mujeres, libro escrito en 1922 y publicado en 1923, encargado y financiado por la SEP. Si bien Mistral precisó en el prólogo que su libro no era una antología, la denominaremos así porque el libro alberga la presencia de 101 autores de diversos lugares y tiempos, en un corpus multifacético escogido por la poeta con relación a las intenciones del libro, es decir, una colección de piezas escogidas dirigidas en específico a la educación literaria de las nińas y mujeres. Por su carácter único en la historia literaria latinoamericana este libro ha recibido atención de investigadoras feministas, pero yo quisiera centrarme en un asunto inexplorado; aventurando la hipótesis de la antología también como una herramienta comunicativa más que a funciones menores o inadvertidas. Creo posible señalar este libro como una carta de presentación de Gabriela Mistral en México, realizada entre otros motivos, para consolidar redes previas, cercanía a autores consagrados y jóvenes incipientes, para visibilizar a los autores que ella consideraba maestros y clásicos, en un intento de construcción y ampliación de canon literario. Sostengo que Mistral incluyó en la selección a escritores mexicanos mayoritariamente vivos, connotados e incipientes figuras del campo literario posrevolucionario -con evidentes diferencias de formación y adhesión al proyecto-, obedeciendo a varias razones. En primer lugar, la autora estaba informada sobre los escritores mexicanos, lo que le ayudó a ubicar a las figuras centrales pudiendo salvar así su condición vulnerable de mujer-escritora dentro de un esquema patriarcal. En segundo lugar, propongo que Lecturas para mujeres fue la forma más efectiva que usó la autora para la creación de trato directo con los mexicanos incluidos, como por ejemplo con Alfonso Reyes. Lecturas para mujeres también funcionó como estrategia de figuración propia, ya que Mistral tenía una importante obra ensayística y poética dispersa en revistas y periódicos.

Para la realización de esta antología contó con el segundo semestre del año 1922 y la ayuda de dactilógrafas y secretarias que debió compatibilizar con sus viajes en comitivas oficiales por los estados de Puebla, Oaxaca, Michoacán y Nuevo León, discursos, 
conferencias, homenajes y constantes visitas a escuelas; la reconstrucción de su itinerario me permite señalar que el tiempo efectivo con el que contó para sentarse con tranquilidad a escribir fue muy poco. Incluyó extractos de obras clásicas y desconocidas, con libertad absoluta de edición, selección y orden del corpus, con el objetivo de darle a las mujeres una parte importante de la cultura artística escrita en la historia "que una mujer debe poseer", advirtiendo que "he procurado compenetrarme de la sensibilidad y el pensamiento mexicanos; no he podido conseguirlo en unos cuantos meses, naturalmente" (Mistral 1923).

He señalado que la poeta llevaba al menos siete años de lecturas de mexicanos y estaba enterada del campo cultural de este país a través de revistas, periódicos e informantes mexicanos residentes en Chile; la enorme calidad y representatividad de su selección evidencia un tiempo de extrema productividad, asunto facilitado por la institucionalidad mexicana mediante la SEP.

Cuadro 2. Autores mexicanos incluidos en Lecturas para mujeres (Ulloa 2019: 133-134).

\begin{tabular}{|c|c|}
\hline Autor/a & Fragmento seleccionado \\
\hline Anónimo & Cantos de Netzahualcóyotl, primer canto \\
\hline Sor Juana Inés de la Cruz & "Soneto" \\
\hline Manuel José Othón & "El perro" \\
\hline Amado Nervo & $\begin{array}{l}\text { "Dar", "Irás por el camino", "Tu Cuerpo", "Se fueron } \\
\text { antes", "Alégrate" y "Muerta" }\end{array}$ \\
\hline Salvador Díaz Mirón & "El fantasma" \\
\hline Luis Gonzaga Urbina & "Así fue..." \\
\hline Enrique González Martínez & $\begin{array}{l}\text { "Eran dos hermanas", "Parábola del huésped sin nombre" } \\
\text { y "Parábola de la Ciega" }\end{array}$ \\
\hline José Juan Tablada & "Los árboles son sagrados" \\
\hline María Enriqueta Camarillo & "Soledad", "Mi carta" y "El afilador" \\
\hline Rafael López & "Bartolomé de las Casas" \\
\hline Carlos Pereyra & "Colón” \\
\hline José Vasconcelos & "El águila y la serpiente" y "Motivos de Cuauhtémoc" \\
\hline Antonio Caso & "La patria dolorosa" \\
\hline Antonio Mediz Bolio & $\begin{array}{l}\text { "La ceiba", "La tortuga", "El girasol” y "El venado y el } \\
\text { faisán" }\end{array}$ \\
\hline Genaro Estrada & "La casa colonial" y "La nao" \\
\hline Alfonso Reyes & "El paisaje de Anáhuac" y "La sonrisa" \\
\hline Julio Torri & "Balada de las hojas más altas" \\
\hline Francisco Monterde García Icazbalceta & "Juana de Asbaje" \\
\hline Josefina Zendejas & "Artista indígena" \\
\hline
\end{tabular}


Solo me detendré en Nervo, Reyes, Camarillo y Zendejas; como sabemos Mistral buscó y reafirmó su vínculo con Nervo durante sus primeros meses de residencia en Ciudad de México; si observamos el Cuadro 2 veremos que la mayor cantidad de fragmentos incluidos en Lecturas para mujeres pertenecen a Nervo.

Una vez que llegó a este país la poeta supo de los escritores que en ese momento estaban ausentes por cumplir funciones diplomáticas representando a México en el exterior: Antonio Mediz Bolio, en Buenos Aires, y Alfonso Reyes, en Madrid. Con Alfonso Reyes comenzó una relación epistolar gracias a esta antología. La primera carta de Mistral a Reyes está fechada en mayo de 1923, en ella la poeta le envía un ejemplar de Lecturas para mujeres seńalando que "hay en esa obra, como Ud. habrá visto, trozos suyos, aprovechados como elemento valioso para formar en las niñas el buen gusto literario" (Vargas 1991: 40), los trozos aludidos son "El paisaje de Anáhuac" y "La sonrisa". También solicita "muy respetuosamente" la autorización de Reyes para enviar a Espańa los libros y la firma de una declaración de licencia para la circulación de estos. El resultado de esta aproximación fue positivo, no solo porque la circulación del libro en España fue favorecida por esta misiva, sino porque Mistral y Reyes trabajaron posteriormente juntos en el Instituto de Cooperación Intelectual (desde 1927) y en la edición de clásicos hispanoamericanos, además de formar una larga e importante amistad que duró décadas.

De María Enriqueta Camarillo, Mistral supo con anticipación:

Hace muchos años, siete o nueve, encontré perdida en una revista femenina la poesía "Así dijo el agua", de María Enriqueta. No conocía ni de referencias a la poetisa; ninguna crítica me había señalado este nombre. La infinita pureza que es el fondo de estas breves estrofas fue como si me lavase en alma enferma. Aquella composición tan perfecta en cuanto a la forma, y tan cabalmente bella, llegó hacerme pensar que la firma oscura fuese un error. Desde entonces, en cada conversación con escritores yo pregunté por ese nombre, hasta que supe que era el de una mujer mexicana, muy divulgado en España, casi desconocido en Chile.

Por medio de González Martínez, ese hombre tan cordial hacia la gloria ajena, tan sin veneno para el camarada de arte, pudimos, mi compañera Laura Rodig y yo, hacerle llegar a España una carta llena de admiración y de simpatía. Y vino en respuesta muy pronto, y siguieron otras y otras cartas, en las cuales fui conociendo el alma del poeta más simple que tal vez tenga nuestra literatura, una mujer muy dama antigua. $Y$ válgame el vocablo, muy hidalga [...]

Los espíritus que crearon mi cariño hacia México, y sobre todo mi confianza hacia el pueblo al cual me entregaría como una familia fueron Amado Nervo, González Martínez y María Enriqueta (Zegers 2007: 73-74).

Es importante señalar que al momento de este escrito Mistral no conocía personalmente a María Enriqueta Camarillo pero aun así fue capaz de escribir sobre ella mencionando su perfil físico y psicológico, haciendo un juicio muy positivo sobre su alma 
y sobre su calidad literaria, tal vez intentando posicionarse como lectora informada y crítica ante la prensa mexicana o tratando de resaltar a otras mujeres -que como ella- debían sortear innumerables cuestionamientos, tratando de apreciar el valor de la escritora pero también de la mujer que debía sufrir las consecuencias de la homosociabilidad literaria que dudaba de la presencia de las mujeres en las letras. De María Enriqueta incluyó los poemas "Soledad" y "El afilador", agregando en una entrevista que gustaría que un mexicano musicalizara sus rondas infantiles y que junto a María Enriqueta Camarillo podría realizar un libro de lecturas femeninas (proyecto que no se concretó).

De Josefina Zendejas incluyó el pequeño fragmento "Artista indígena" que formaba parte del libro Gusanito: Poemas en prosa dedicados a los niños de la América, editado por Cvltvra (editorial amiga de la red vasconcelista) en 1923, mismo ańo de Lecturas para mujeres. Aun no me es posible acreditar que Mistral conoció y frecuentó a Zendejas, pero sí puedo señalar que estaba muy enterada de su obra, seguramente Gusanito aún no había sido publicado cuando Mistral lo incluyó, ya que Lecturas para mujeres se terminó de imprimir el 31 de enero de 1923 y Gusanito el 20 de abril del mismo año. Lo importante es que Gusanito había sido comentado por Juana de Ibarbourou, poeta que también fue intelectual pública, por quien Mistral sentía admiración y mantenía una larga relación epistolar. Ibarbourou envió una carta a Zendejas en donde comentaba la obra:

Es un encanto, de verso, su libro GUSANITO. Me ha sorprendido y emocionado tanta comprensibilidad del alma y el lenguaje infantil; tanta gracia, tanta frescura, tanta ingenuidad y el perfecto con gusto y equilibrio con que está realizada la obra, sin caer en niñerismos absurdos. Hondamente mujer y hondamente maestra se ve que es usted. Yo la felicito y le auguro un éxito franco y bellísima con su precioso gusanito. Le prometo espontáneamente, escribir sobre él, MI PEQUEÑA TAGORE (Zendejas 1923: 1).

La inclusión de Zendejas en Lecturas para mujeres sirve para señalar que Mistral fue una intelectual extremadamente conectada con sus pares, que sí supo conocer a sus contemporáneas.

\section{Conclusiones}

Es difícil señalar aquí la enorme cantidad de archivos históricos consultados para elaborar esta cronología y relación de publicaciones y vínculos; queda pendiente una interpretación mayor a la luz de otras investigaciones. Este artículo solo indaga en el cómo se produjo el acercamiento de la escritora chilena con sus pares mexicanos recalcando los mecanismos de acercamiento bibliográfico e ideológico.

Mistral conoció, durante su estadía en Ciudad de México, a los editores de sus primeros libros, consiguiendo además ser incluida en antologías de España y Estados 
Unidos, donde frecuentó a destacados escritores, intelectuales y políticos latinoamericanos como Rafael Heliodoro Valle, Alfredo Palacios o Víctor Raúl Haya de la Torre. En esa ciudad su hábitus estuvo fuertemente impactado por quien se convertiría en su máxima aliada y maestra sobre México; Palma Guillén la primera mujer embajadora en Latinoamérica quien fuera su pareja sentimental por más de una década, e inició amistad con escritores como Alfonso Reyes, gran amigo y cómplice, solo por mencionar aspectos centrales de su profesionalización e internacionalización ocurrida en este periodo. Mistral entonces fue a México por voluntad propia y por una afortunada coincidencia entre sus ideas renovadoras y el ambiente posrevolucionario; lo pudo realizar gracias a sus habilidades relacionales, pero también gracias a una experiencia madurada con una notable formación autodidacta como maestra, intelectual, representante diplomática y escritora, cuatro facetas distintas que ella desarrolló simultáneamente.

\section{Obras CITADas}

Bourdieu, Pierre. 2002. Campo de poder, campo intelectual. Itinerario de un concepto. Buenos Aires: Montressor.

Cabello, Claudia. 2018. Artesana de sí misma. Gabriela Mistral una intelectual en cuerpo y palabra. West Lafayette: Purdue University Press.

Fell, Claude. 1989. José Vasconcelos: Los años del águila (1920-1925). Educación, cultura e iberoamericanismo en el México posrevolucionario. Ciudad de México: Instituto de Investigaciones Históricas, UNAM.

Horan, Elizabeth. 2013. Motivos. The life of St. Francis. Tempe, Arizona: Bilingual Press/ Editorial bilingüe.

Loveluck, Juan. 1970. "Cartas de Gabriela Mistral a Amado Nervo". Revista Iberoamericana 36.72: 495-508.

Luigi, Raffaele. 2016. José Enrique Rodó en México. Tesis para optar al grado de Doctor en Letras: Universidad Nacional Autónoma de México.

Martínez, Leonardo. 2002. Alfonso Reyes/ Enrique González Martínez: El tiempo de los patriarcas. Epistolario 1909-1952. Ciudad de México: FCE.

Mistral, Gabriela. 1923. Lecturas para mujeres. Ciudad de México: SEP. . 1920. 17 de septiembre. "Carta a Maximiliano Salas Marchán”. Biblioteca Nacional de Chile: Archivo del Escritor.

Palou, Pedro. 1997. La casa del silencio: aproximaciones en tres tiempos a Contemporáneos. Zamora, Morelia: El Colegio de Michoacán.

Quezada, Jaime. 2002. Bendita mi lengua sea. Diario intimo de Gabriela Mistral (19051956). Santiago: Planeta.

Sánchez-Prado, Ignacio. 2006. Naciones intelectuales: La modernidad literaria mexicana de la constitución a la frontera (1917-2000). Tesis para optar al grado de Ph.D. in Hispanic Languages and Literatures: Universidad de Pittsburgh. 
Ulloa, Carla. 2019. La construcción de una intelectual: Gabriela Mistral en el campo cultural mexicano 1922-1924. Tesis para optar al grado de Doctora en Estudios Latinoamericanos: Universidad Nacional Autónoma de México. . 2017. "Poder, cultura y saber. Una pregunta por las intelectuales. Gabriela Mistral en México 1922-1924”. En Blazquez, Norma y Patricia Castañeda. Lecturas críticas en investigación feminista. UNAM: 173-193.

Vargas, Luis. 1991. Tan de usted. Epistolario de Gabriela Mistral con Alfonso Reyes. Santiago: Ediciones Universidad Católica de Chile.

Vasconcelos, José. 1968. El desastre. Ciudad de México: Jus S. A.

Zegers, Pedro. 2007. Gabriela y México. Santiago: RIL Editores.

Zendejas, Josefina. 1923. Gusanito: Poemas en prosa dedicados a los niños de la América. Ciudad de México: Editorial Cvltvra.

\section{REVISTAS}

El Libro y El Pueblo: Revista Mensual Bibliográfica del Departamento de Bibliotecas de la SEP, México, 1922.

El Maestro: Revista de Cultura Nacional, México 1921-1923.

México Moderno. Revista mensual de Letras y Arte, México, 1920-1922.

Pegaso. Revista semanal 16. México, 1917.

Repertorio Americano. Costa Rica, 1921-1924.

Revista de Revistas. México, 1921-1922. 